Nervenarzt 2009 [Suppl 1] $\cdot 80: 3-3$

DOI 10.1007/s00115-009-2748-7

(C) Springer Medizin Verlag 2009

\section{J. Czekalla}

Neurologie, Immunologie u. Ophthalmologie, Bayer Vital, Division Bayer Schering Pharma, Leverkusen

\title{
1. Forschungswerkstatt Multiple Sklerose
}

\section{Präsentation einer Vielfalt innovativer Studien von der Neuroimmunologie bis zur Bildgebung}

Nachdem das Konzept der Forschungswerkstatt mit den Themenschwerpunkten Infektiologie und Kardiologie bei der Bayer Vital GmbH, Bayer Schering Pharma, bereits seit nahezu 10 Jahren Tradition besitzt, wurde im November 2008 für den Bereich Neurologie/Immunologie erstmals eine Forschungswerkstatt Multiple Sklerose (MS) ins Leben gerufen, die mit großer Beteiligung führender Forschungsgruppen im Herzen der Bayer AG in Leverkusen stattfand.

Im Mittelpunkt der insgesamt 35 wissenschaftlichen Vorträge standen aktuelle und größtenteils noch nicht publizierte Ergebnisse aus Studien zu Interferon $\beta-1 b$ (Betaferon ${ }^{\circledR}$ ). Durch die verschiedenen Themenblöcke führten die Vorsitzenden Prof. Dr. med. Ralf Gold, Bochum, Prof. Dr. med. Hans-Peter Hartung, Düsseldorf, Prof. Dr. med. Bernhard Hemmer, München, und Prof. Dr. med. Patrick Oschmann, Bayreuth. Präsentiert und diskutiert wurden immunologische und neuroprotektive Effekte von Interferon $\beta$-1b, Fortschritte bei Biomarkern und Diagnostik der MS sowie neuropsychologische und andere klinische Aspekte der MS-Therapie. Aktuelle Daten zur Frühtherapie (5-Jahres-Daten der BENEFIT-Studie) sowie ein Ausblick auf Alemtuzumab (Genzyme/Bayer Schering Pharma) als mögliche zukünftige Therapieoption aus der MS-Entwicklungspipeline in Phase III rundeten das breitgefächerte Programm ab.

Ich möchte mich an dieser Stelle bei allen Teilnehmern für die gelungene Veranstaltung bedanken, bei den Vorsitzenden für ihre engagierte Leitung durch die ein- zelnen Themenblöcke, bei allen Vortragenden für ihre spannenden und innovativen Forschungsergebnisse und Referate, beim Auditorium für die angeregte und hochwertige Diskussion sowie nicht zuletzt auch bei den Mitarbeitern der Bayer Vital GmbH, die für die Konzeption und den reibungslosen Ablauf der Forschungswerkstatt verantwortlich waren.

Die 1. Forschungswerkstatt MS hat gezeigt, dass fast 20 Jahre nach der ersten klinischen Anwendung von Interferon $\beta$ $1 \mathrm{~b}$ immer noch weitere immunologische Wirkmechanismen dieses facettenreichen Proteins erforscht werden. Parallel dazu verbessern zunehmend sensitivere elektrophysiologische und bildgebende Verfahren die Diagnostik der MS und damit die Aussage über Prognose und Verlauf der Erkrankung, wie zum Beispiel innovative Methoden zur Quantifizierung von Hirnatrophie, das Diffusions Tensor Imaging (DTI) oder neue Läsionskontrastmittel wie USPIO aus der Bayer-Schering-Pharma-Forschung. Nicht zuletzt sind all diese Ansätze auch eine entscheidende Vorraussetzung für die Verbesserung der Lebensqualität von MS-Patienten, deren Wechselspiel mit wichtigen Begleitsymptomen der Erkrankung, wie Fatigue, Depression und Kognitionsstörungen, zunehmend im Fokus neuer Studien steht.

Als größtes deutsches Pharmaunternehmen unterstützt Bayer Schering Pharma sowohl die Grundlagenforschung als auch klinische Studien und bietet ambitionierten Wissenschaftlern mit der Forschungswerkstatt auch in Zukunft eine Plattform für den Dialog. Durch die Kooperation von klinischer Forschung und dem Entwicklungsbereich für radiologische und molekulare Bildgebung ergeben sich neue Chancen zur Verbesserung der Diagnostik und Therapie neurodegenerativer und neuroinflammatorischer Erkrankungen wie MS und Alzheimer-Demenz. Des Weiteren investieren wir auch zukünftig in Studien, die aufzeigen, wie die Anwendung von Betaferon $^{\circledR}$ verbessert werden kann, zum Beispiel durch Patientenbetreuungsprogramme, neue Applikationssysteme sowie innovative Technologien zur Adhärenzsteigerung.

Das vorliegende Supplement ermöglicht Ihnen einen spannenden Überblick über den aktuellen Stand der präsentierten Studien in Form von „extended Abstracts“. Mit großem Interesse sehe ich den weiterführenden Vollpublikationen der einzelnen Studien entgegen sowie der 2. Forschungswerkstatt MS im Jahr 2010.

Dr. med. Jörg Czekalla

Leiter Medizin, Neurologie, Immunologie und Ophthalmologie, Bayer Vital, Division Bayer Schering Pharma, Leverkusen

\section{Korrespondenzadresse Dr. med. J. Czekalla

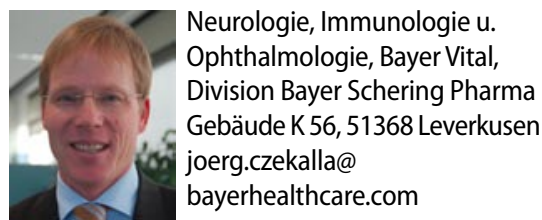

Interessenkonflikt. Der korrespondierende Autor gibt an, dass er Mitarbeiter der Bayer Schering Pharma AG ist. 